\title{
Variation in Sleep Pattern Due to Lockdown among Collegiate Students
}

\author{
Manisha Bhandari ${ }^{1}$, Rabab Kaur ${ }^{2}$ \\ ${ }^{1}$ Student, Galgotias University, ${ }^{2}$ Assistant Professor, Galgotias University, Plot No. 2, Yamuna Expy, \\ Opposite, Buddha International Circuit, Sector 17A, Greater Noida, Uttar Pradesh 203201
}

\begin{abstract}
Background: Due to the vast spread of corona virus, lockdown was announced in many countries, and hence many people are facing psychological, physical, economic and social changes. Students at the college experience a variety of sleep problems which can have a direct effect on academic success, health and mood. Sleep disruption and excessive daytime sleep are common problems for students.

Subject and Method: 141 college students between the age of 17 to 24 were taken from various colleges. The structured Pittsburgh Sleep Quality Index was used and was circulated amongst students through Google forms and the sleep log, and demographic sheet was used to test sleep length, daytime sleepiness and related sleep issues.
\end{abstract}

Study Design: A cross sectional study.

Results: The result, on the basis of the sleep pattern of college students, was classified into 5 categories: (healthy sleep pattern, mild, moderate, severe and worst). 53.90\% out of 141 respondents o had healthy sleep pattern range (0-5), 39.71\% had mild sleep pattern range (6-10), $4.96 \%$ out of 141 respondents had moderate sleep pattern range (11-15), and $1.41 \%$ out of 141 respondents had severe sleep pattern range (16-20).

Conclusion: In our study we found that during the lockdown majority of college students had a healthy sleep pattern and least of the college students had a severe sleep pattern range.

Keywords: Pittsburgh Sleep Quality Index, Coronavirus, Lockdown, sleep pattern, college students.

\section{Introduction}

Sleep is a physiological process that can alternate with internal and external stimulation between conscious stage and unconscious stage ${ }^{1}$. Sleep absorbs about one third of our lives and thus it plays a crucial role which is key to emotional, physical development and stability. A good sleep is needed for better growth and stability, if there is lack of sleep than it may decrease physical and mental capacities along with mental problem such as anxiety and depression, therefore sleep loss has tremendous impact on both society as well as individuals ${ }^{2}$. Now days the second most common symptom of mental distress is commonly known as sleep disturbance, according to many surveys now days one third of college students reports difficultly in sleeping ${ }^{3}$. Now days insufficient sleep and irregular sleep wake schedule among the college student are becoming major health problem, which is directly related to decrease the quality of life, due to the hectic schedules college student suffer from sleep disturbance, fatigue and mood change ${ }^{4}$. Many research show that there is high chance of sleep disturbance in college student than the general population $^{5}$.

Sleep disturbance for college students is directly associated with cognition, academic performance, emotional, intelligence, psychological and physical health. Still no research is available on the impact of sleep quality on college student's health and well-being. The main factor for sleep disturbance to the college students are all nightery parties, late-night studying and social obligation, work and alcohol/or drug abuse ${ }^{8}$. It's hard to fall asleep earlier in the evening for the college 
students because they cannot wake up early. In addition, the college students sleep pattern is characterized by inadequate length of sleep, delayed onset of sleep and frequency of napping during the day that directly affect the study ${ }^{9}$.

The world health organization (WHO) confirmed in January 2020 the outbreak of a new corona disease, Covid-19 which had already reported that there is a potential high risk of covid-19 spreading to the entire world ${ }^{10}$. As a consequence of the spread of covid-19 disease, almost all countries form formulated the guidelines which include isolating, lockdown quarantining and social distancing at these times. Lockdown is the concept used for separation, or restriction of movement of the community during an epidemic of infectious disease. Because of a dramatic rise in covid19 incidents the government imposed an immediate lockdown to prevent the disease from spreading across the population ${ }^{11}$. Nowadays, the current situation is facing negative effect on many factors which influence sleep quality and almost all people are experiencing major changes in their daily life ${ }^{12}$. In lockdown college students increased the use of digital media to alter sleep habits at bedtime. The increased sleep pattern leads to higher levels of depression, anxiety, and stress in many people ${ }^{13}$. Sleep issue can be significant concern for all during the lockdown, but some individuals are at higher risk of experiencing sleep problems than others. Women experience poor sleep and insomnia more often than men. In addition, students can depend on their phone with the likelihood of mobile phones, which directly affects the sleep pattern. According to the national sleep foundation in the U.S. 2011 task force, 50 percent of young adults use their cell phones and the internet before they sleep. The improper use of computers and mobile devices in the bedroom thus delays bedtime and the raise time ${ }^{14}$.

\section{Methodology}

This chapter discusses the process used for the analysis which provides explanation of the topics used in data collection and analysis and procedures. The research Sample consisted of 141 College Students based on the structured questionnaire respondents.

The subject of this study was 141 college students. The primary data was collected from college students by using stratified random sampling method. Universe of this present study was the college students in lockdown, India . Besides that, all of them were between 17 to 24 years of age. The data has been collected through college students by structured questionnaires using online Google form.

\section{Inclusion Criteria:}

1. Gender- male and female

2. College students

3. Subject should be between 17 to 24 years

4. Able to understand Hindi and English language

5. Individuals who were cooperative

6. Young healthy individuals

\section{Exclusion Criteria:}

1. Students with neurological issued

2. Non-cooperative subjects

\section{Result}

In this survey study 141 subjects participated and answered all the questions of sleep quality questionnaire and also provided detailed demographic information including name, age, gender, contact number, address, and contact details for email.

The Pittsburgh sleep quality index (PSQI) was used. Table 1 shows subjective sleep quality and according to it $32.6 \%$ out of 141 respondents, sleep quality rate is very good, $48.9 \%$ out of 141 respondents, sleep quality rate is fairly good, $13.4 \%$ out of 141 respondents, sleep quality rate is fairly bad and $5 \%$ out of 141 respondents, sleep quality rate is very bad. According to table $230.4 \%$ out of 141 respondents took less than 15 minutes to sleep at night, $42.5 \%$ out of 141 respondents took $16-30$ minutes to sleep at night, $14.1 \%$ out of 141 respondents took $31-60$ minutes to sleep at night and $12.7 \%$ out of 141 respondents took more than 60 minutes to sleep at night. Table 2.1 shows that $56.7 \%$ out of 141 respondents had trouble sleeping in the past month because they could not sleep within 30 minutes, $17.02 \%$ out of 141 respondents had trouble sleeping less than once a week because they could not get sleep within 30 minutes, $14.8 \%$ out of 141 respondents had trouble sleeping once or twice a week because they could not get sleep within 30 minutes and $11.3 \%$ out of 141 respondents had trouble sleeping three or more times a week because they could not sleep within 30 minutes.

In table 2 and figure 2 we calculated the component in which we analyzed the responses of question 2 and $5 \mathrm{a}$. 
For component 3, $343.9 \%$ out of 141 respondents got 7 hours or more hours of actual sleep at night, $48.3 \%$ out of 141 respondents got 6 to 7 hours of actual sleep at night. $5.6 \%$ out of 141 respondents got 5 to 6 hours of actual sleep at night and $2.1 \%$ out of 141 respondents received 5 hours of real night-time sleep. For component 4, sleep effectiveness of sleep is determined by using formula (hours slept $\div$ hours in bed) $\times 100$. For component 5, $14.1 \%$ out of 141 respondents had sleep disturbance during the past month, $69.9 \%$ out of 141 respondents had sleep disturbance less than once a week, $16.3 \%$ out of 141 respondents had sleep disturbance once or twice a week and $0 \%$ out of 141 respondents had sleep disruption three times or more.

The component 6 shows that $83.6 \%$ out of 141 respondents have taken sleep medicine during past month, $9.21 \%$ out of 141 took sleep medicine less than once a week, $6.38 \%$ out of 141 took sleep medicine once or twice a week and $0.70 \%$ out of 141 took sleep medicine three or more times a week. For component 7 , $64.5 \%$ out of 141 respondents had trouble staying awake in the last month, $19.1 \%$ out of 141 respondents had trouble staying awake less than once a week, $12.7 \%$ out of 141 respondents had trouble staying awake less than once or twice a week and $3.5 \%$ out of 141 respondents had difficulty staying awake three or more days a week. Table 7.1 reveals that $51.0 \%$ out of 141 respondents had no issue at all in maintaining enthusiasm for doing things, $29.7 \%$ out of 141 respondents had only a very slight problem, $16.3 \%$ out of 141 respondents had somewhat of a problem and $2.8 \%$ out of 141 respondents had a very major problem.

Table 1: Quality of Subjective sleep and Latency sleep

\begin{tabular}{|c|c|c|c|}
\hline Frequency (N) & Percentage & Sleep Quality Response & Component Score \\
\hline 46 & $32.6 \%$ & Very good & 0 \\
\hline 69 & $48.9 \%$ & Fairly good & 2 \\
\hline 19 & $13.4 \%$ & Fairly bad & 3 \\
\hline 7 & $5 \%$ & Very bad & Component score \\
\hline Frequency (N) & Percentage \% & $\leq 15$ minutes & 0 \\
\hline 43 & $30.4 \%$ & $16-30$ minutes & 2 \\
\hline 20 & $42.5 \%$ & $31-60$ minutes & 3 \\
\hline
\end{tabular}

According to Table 1: $32.6 \%$ out of 141 respondents, sleep quality rate is very good, $48.9 \%$ out of 141 respondents sleep quality rate is fairly good, $13.4 \%$ out of 141 respondents sleep quality rate is fairly bad and $5 \%$ out of 141 respondents sleep quality response, the percentage is very low.
$30.4 \%$ out of 141 respondents take time to sleep at night in $\leq 15$ minutes, $42.5 \%$ out of 141 respondents take time to sleep at night in 16 -30 minutes, $14.1 \%$ out of 141 respondents take time to sleep at night in 31-60 minutes, $12.7 \%$ out of 141 respondents take time to sleep at night in $>60$ minutes.

Table 2: Duration of sleep

How many hours you have got to sleep at night during the last month?

\begin{tabular}{|c|c|c|c|}
\hline Frequency & Percentage \% & Sleep duration & Component score 3 \\
\hline 62 & $43.9 \%$ & $\geq 7$ hours & 0 \\
\hline 68 & $48.3 \%$ & $6-7$ hours & 2 \\
\hline 8 & $5.6 \%$ & $5-6$ hours & 3 \\
\hline
\end{tabular}


According to Table 2: Among the 141 responses, $43.9 \%$ receive 7 hours among actual night-time sleep, $48.3 \%$ receive 6 to 7 hours of actual night-time sleep. Among the 141 responses, $5.6 \%$ receive 5 to 6 hours among real sleep at night and $2.1 \%$ receive 5 hours of actual night-time sleep.

Table 3: Sleep efficiency

\begin{tabular}{|c|c|c|}
\hline Frequency (N) & Sleep efficiency & Component 4 score \\
\hline 101 & $>85 \%$ & 0 \\
\hline 15 & $75-84 \%$ & 1 \\
\hline 16 & $65-74 \%$ & 2 \\
\hline 9 & $<65 \%$ & 3 \\
\hline
\end{tabular}

According to Table 3 sleep efficiency is calculated by using formula (hours slept $\div$ hours in bed) $\times 100$

Table 4: Sleep disorder and use of medication for sleep

\begin{tabular}{|c|c|c|c|}
\hline Frequency & Percentage & Sleep disturbance & Component 5 score \\
\hline 20 & $14.1 \%$ & Not during pass month & 0 \\
\hline 98 & $69.9 \%$ & Less than once in a week & 1 \\
\hline 23 & $16.3 \%$ & Twice or once a week & 3 \\
\hline 0 & 0 & Three or more times per week & Component Score \\
\hline Frequency (N) & Percentage $\%$ & Use of medication for sleep & 0 \\
\hline 118 & $83.6 \%$ & Not in the past month & 1 \\
\hline 13 & 9.21 & Less than one week & 2 \\
\hline
\end{tabular}

According to Table 4: $14.1 \%$ have sleep disturbance not during pass month, $69.9 \%$ out of have sleep disturbance less than once a week, $16.3 \%$ out of have sleep disturbance once or twice a week and $0 \%$ out have sleep disturbance have occurred three or more times per a week.
83.6\% have taken sleep medicine not during past month, $9.21 \%$ have taken sleep medicine less than once a week, $6.38 \%$ have taken sleep medicine once or twice a week and $0.70 \%$ have taken sleep medicine three or more times per week.

Table 5: Dysfunction on the day

\begin{tabular}{|c|c|c|c|}
\hline Frequency (N) & Percentage \% & $\begin{array}{c}\text { Over the past few month, how often have you had trouble } \\
\text { staying awake while driving, eating, or socializing? }\end{array}$ & Score \\
\hline 91 & $64.5 \%$ & Not during the last month & 0 \\
\hline 27 & $19.1 \%$ & Less than one week & 1 \\
\hline 18 & $12.7 \%$ & Once or two times a week & 2 \\
\hline 5 & $3.5 \%$ & Three or more times per week & 3 \\
\hline
\end{tabular}

According to Table 5: $64.5 \%$ have trouble staying awake not during the past month, $19.1 \%$ have trouble staying awake less than once a week, $12.7 \%$ have trouble staying awake once or two times a week and 3.5\% have trouble staying up three or more times per week. 
Global PSQI Score: A total of seven components

Table 6

\begin{tabular}{|c|c|c|c|}
\hline Frequency (N) & Percentage \% & Range (0-21) & Sleep Quality \\
\hline 76 & $53.90 \%$ & $0-5$ & Healthy \\
\hline 56 & $39.71 \%$ & $6-10$ & Mild \\
\hline 7 & $4.96 \%$ & $11-15$ & Moderate \\
\hline 2 & $1.41 \%$ & $16-20$ & Severe \\
\hline 0 & $0 \%$ & $21-25$ & Worst \\
\hline
\end{tabular}

According to the global PSQI score: $53.90 \%$ have healthy sleep quality,39.71\% have mild sleep quality, $4.96 \%$ have moderate sleep quality and $1.41 \%$ have severe sleep quality.

\section{Result}

Global PSQI Score: Sum of seven components: According to the global PSQI score 53.90\% out of 141 respondents of college student have healthy sleep pattern, $39.71 \%$ have mild sleep pattern, $4.96 \%$ have moderate sleep pattern and $1.41 \%$ have severe sleep pattern.

\section{Discussion}

We investigated variation of the college students sleep pattern during lockdown in cross- sectional study. As expected, during lockdown college students sleep pattern may be changed. The information of the results must consider the limitations of the study mainly depend on self- reports regarding sleep schedule. The COVID-19 pandemic has created damaged millions of people to live under strict blackout conditions and people who go to bed and wake up late, spend more time in bed and record a poorer standard of sleep as well. Decreased sleep quality was stronger with high levels of depression, anxiety, and stress. Loss of sleep among college students poses a serious risk to academic performance, both physical and emotional health. For the study, a total of 141 college students were chosen, they were evaluated for the parameters of inclusion and exclusion criteria. College student sleep pattern is tested on the basis of the result of PSQI they were put under the five groups namely 1) Good Sleep quality, 2) Mild Sleep quality,3) moderate Sleep quality, 4) Extreme Sleep quality, and 5) Worst Sleep quality.

In our study one questionnaire is used to determine the sleep pattern of college students during lockdown.
The questionnaire instruction analyzed the results. There are seven component scores in the scoring PSQI, each score 0 (no difficulty) to 3 (severe difficulty). Scores of the components are summed up to generate a global score ( 0 to 21$)$ higher score mean poorer quality of sleep. Good and satisfied sleep is not only central to sound body health, but is also of primary importance to cognitive and social activities. Learning plays a significant part in learning and memory process for the students.

In our analysis we identified the characteristics of socio-demographic and lifestyle, nighttime behaviors and sleep habits and their results. Besides low sleep quality students also showed poor quality of sleep. Table 1 reveals that $32.6 \%$ out of 141 response of college have very good sleep pattern and 5\% out of 141 responses have very disturbed sleep pattern. Poor sleep quality has been associated with negative moods reported to be significantly higher by themselves. Table 2 shows $42.5 \%$ out of 141 response of college student take 16 to 30 -minute times to sleep at nights. $12.7 \%$ out of 141 response take more than 60 minutes to sleep at night so we should stick to the same sleep schedule and wake up time even on the weekends, so that it can help us sleep at right time. Comparing the previous study, we found that $43.9 \%$ had more than 7 hours of real night sleep and $16.3 \%$ had once or twice a week disrupted sleep. Sleep medicine is taken once or twice a week by $6.38 \%$ students.

According to this research we found global PSQI score is used to sum the seven components. In the current study, data shows that during lockdown majority of the college student had healthy sleep pattern $(53.90 \%)$ range of the PSQI ( $0-5), 39.71 \%$ had mild sleep pattern range of the PSQI (6-10), 4.96\% had moderate sleep pattern range of the PSQI (11-15) and $1.41 \%$ had severe sleep pattern range of the PSQI (16-20). 


\section{Limitations of the study:}

- One of the limitations of this study is small sample size, study was of short duration, the interval between the age group is less and study is done on young healthy individuals only.

- Another drawback was that this analysis consisted of a one- time study, so it is difficult to determine the connection between sleep disturbance and poor sleep quality.

\section{Recommendations:}

- This analysis can be carried out on a larger sample size.

- The period of research can be longer.

- There may be variations in the age group used for study.

\section{Conclusion}

We assume there is need for more research into sleep cycle and their effect on the working. There should be a study of students with good sleep habits. Sleep requirements differ according to individual needs. Some need more than 7 hours of effective sleep, other may need as little as 5 hours of sleep. Further research can identify factors in lifestyle which promote good quality of sleep.

In the current study we found that during lockdown period most students had a healthy sleep pattern. Sleep disorder can have a huge effect on people lives. These findings support that there is no variation of sleep pattern during lockdown. Healthy sleep pattern decreases the anxiety, tension, depression and stress .

Ethical Clearance: Taken from University Ethical Committee

\section{Source of Funding: Self}

\section{Conflict of Interest: Nil}

\section{References}

1. OrsalO, Orsal O, Alparslan GB, Unsal A. Evaluation of the relation between quality of sleep and anxiety among university students. HealthMED. 2012 Jan 1;6(7):2244-55.

2. Ban DJ, Lee TJ. Sleep duration, subjective sleep disturbances and associated factors among university students in Korea. Journal of Korean
Medical Science. 2001 Aug; 16(4):475.

3. Heath AC, Eaves LJ, Kirk KM, Martin NG. Effects of lifestyle, personality, symptoms of anxiety and depression, and genetic predisposition on subjective sleep disturbance and sleep pattern. Twin Research and Human Genetics. 1998 Aug;1(4):176-88.

4. Singh R, Sharma R, Suri JC, Das S. Impact of sleep patterns on mood and academic performance of medical students. Indian Journal of Sleep Medicine (IJSM). 2009;4(2).

5. Jenkins SM. Sleep habits and patterns of college students and their relationship to selected personality characteristics

6. MAHRAN DG, AHMED SM. Sleep Disorders among Female Students in Assiut University, Upper Egypt. Med. J. Cairo Univ. 2012;83(2):17985.

7. Ishita M, Kuldeep S. Impact of Digital Media on Sleep Pattern Disturbance in Medical and Nursing students. Science [ETEBMS-2016]. 2016 Mar;5(6).

8. Kaur G, Sharma V, Singh A. Association of sleep quality with general health: an Indian college students' study. Int J Med Sci Public Health. 2015 Dec 1;4(12):1767

9. Lowry M, Dean K, Manders K. The link between sleep quantity and academic performance for the college student. Sentience. 2010;3(2):16-9

10. Anjum A, Bajwa MA, Saeed R. SLEEP PATTERNS. The Professional Medical Journal. 2014;21(01):148-56.

11. World Health Organization. Mental health and psychosocial considerations during the COVID-19 outbreak, 18 March 2020. World Health Organization; 2020.

12. Altena E, Baglioni C, Espie CA, Ellis J, Gavriloff D, Holzinger B, Schlarb A, Frase L, Jernelöv S, Riemann D. Dealing with sleep problems during home confinement due to the COVID-19 outbreak: practical recommendations from a task force of the European CBT-I Academy. Journal of Sleep Research. 2020 Apr 4.

13. Lund HG, Reider BD, Whiting AB, Prichard JR. Sleep patterns and predictors of disturbed sleep in a large population of college students. Journal of adolescent health. 2010 Feb 1;46(2):124-32.

14. Cellini N, Canale N, Mioni G, Costa S. Changes in sleep pattern, sense of time, and digital media use during COVID-19 lockdown in Italy. 
15. Saha S, Dutta T. A STUDY ON THE PSYCHOLOGICAL CRISIS DURING THE LOCKDOWN CAUSED DUE TO COVID-19 PANDEMIC.

16. Albhlal LA, Alanzi FG, Ghannam KB, Alqahtani A, Alenazi AQ, Alanazi MQ. Sleep Disturbance
Patterns among Medical Students, Saudi Arabia. Arch Med. 2017;9:3.

17. BuboltzJr WC, Brown F, Soper B. Sleep habits and patterns of college students: a preliminary study. Journal of American college health. 2001 Nov $1 ; 50(3): 131-5$. 